\title{
LIPSCHITZ SPACES AND BOUNDED MEAN OSCILLATION OF HARMONIC MAPPINGS
}

\author{
SH. CHEN, S. PONNUSAMY ${ }^{凶}$, M. VUORINEN and X. WANG \\ (Received 16 July 2012; accepted 18 October 2012; first published online 18 January 2013)
}

\begin{abstract}
We first study the bounded mean oscillation of planar harmonic mappings. Then we establish a relationship between Lipschitz-type spaces and equivalent modulus of real harmonic mappings. Finally, we obtain sharp estimates on the Lipschitz number of planar harmonic mappings in terms of the bounded mean oscillation norm, which shows that the harmonic Bloch space is isomorphic to $\mathrm{BMO}_{2}$ as a Banach space.
\end{abstract}

2010 Mathematics subject classification: primary 30H10, 30H30; secondary 30C20, 30C45.

Keywords and phrases: harmonic mapping, majorant, Lipschitz space, $B M O_{p}$, equivalent modulus, harmonic Bloch space, Green's theorem.

\section{Introduction and main results}

Let $\mathbb{C}$ denote the complex plane. For $a \in \mathbb{C}$, let $\mathbb{D}(a, r)=\{z:|z-a|<r\}$. In particular, we use $\mathbb{D}_{r}$ to denote the disc $\mathbb{D}(0, r)$ and $\mathbb{D}$ the unit disc $\mathbb{D}_{1}$. A complex-valued function $f$ defined on $\mathbb{D}$ is called harmonic in $\mathbb{D}$ if and only if both the real and imaginary parts of $f$ are real harmonic in $\mathbb{D}$. It is known that every harmonic mapping $f$ defined in $\mathbb{D}$ admits a decomposition $f=h+\bar{g}$, where $h$ and $g$ are analytic in $\mathbb{D}$. We refer to $[10,12,13,19,34]$ for the theory of planar harmonic mappings. For harmonic mappings $f$ defined on $\mathbb{D}$, we use the following standard notation:

$$
\Lambda_{f}(z)=\max _{0 \leq \theta \leq 2 \pi}\left|f_{z}(z)+e^{-2 i \theta} f_{\bar{z}}(z)\right|=\left|f_{z}(z)\right|+\left|f_{\bar{z}}(z)\right|
$$

and

$$
\lambda_{f}(z)=\min _{0 \leq \theta \leq 2 \pi}\left|f_{z}(z)+e^{-2 i \theta} f_{\bar{z}}(z)\right|=\left\|f_{z}(z)|-| f_{\bar{z}}(z)\right\| .
$$

A continuous increasing function $\omega:[0, \infty) \rightarrow[0, \infty)$ with $\omega(0)=0$ is called a majorant if $\omega(t) / t$ is nonincreasing for $t>0$ (see $[14,28]$ ). Given a subset $\Omega$ of $\mathbb{C}$, a

The research of Matti Vuorinen was supported by the Academy of Finland, Project 2600066611. The research of Sh. Chen and X. Wang was partly supported by NSF of China (No. 11071063). The authors wish to express their gratitude to Professor Miroslav Pavlović for valuable comments. The second author is currently on leave from the Indian Institute of Technology, Chennai, India.

(C) 2013 Australian Mathematical Publishing Association Inc. 0004-9727/2013 \$16.00 
function $f: \Omega \rightarrow \mathbb{C}$ is said to belong to the Lipschitz space $L_{\omega}(\Omega)$ if there is a positive constant $M$ such that

$$
|f(z)-f(w)| \leq M \omega(|z-w|) \quad \text { for all } z, w \in \Omega .
$$

For $\delta_{0}>0$ and $0<\delta<\delta_{0}$, we consider the following conditions on a majorant $\omega$ :

$$
\int_{0}^{\delta} \frac{\omega(t)}{t} d t \leq M \omega(\delta)
$$

and

$$
\delta \int_{\delta}^{+\infty} \frac{\omega(t)}{t^{2}} d t \leq M \omega(\delta),
$$

where $M$ denotes a positive constant. A majorant $\omega$ is said to be regular if it satisfies (1.2) and (1.3) (see [14, 28]).

Dyakonov [14] discussed the relationship between Lipschitz space and bounded mean oscillation on holomorphic functions in $\mathbb{D}$, and obtained the following result. In order to state Theorem A, we first introduce some notation. Let $G$ be a domain of $\mathbb{C}$. We use $d_{G}(z)$ to denote the Euclidean distance from $z$ to the boundary $\partial G$ of $G$. In particular, we always use $d(z)$ to denote the Euclidean distance from $z$ to the boundary of $\mathbb{D}$.

Theorem A [14, Theorem 1]. Suppose that $f$ is a holomorphic function in $\mathbb{D}$ which is continuous up to the boundary of $\mathbb{D}$. If $\omega$ and $\omega^{2}$ are regular majorants, then

$$
f \in L_{\omega}(\mathbb{D}) \Longleftrightarrow P_{|f|^{2}}(z)-|f(z)|^{2} \leq M \omega^{2}(d(z)),
$$

where

$$
P_{|f|^{2}}(z)=\frac{1}{2 \pi} \int_{0}^{2 \pi} \frac{1-|z|^{2}}{\left|z-e^{i \theta}\right|^{2}}\left|f\left(e^{i \theta}\right)\right|^{2} d \theta .
$$

The following result is an analogue of Theorem A for planar harmonic mappings.

Theorem 1.1. Suppose that $\omega$ is a majorant and that $f$ is a harmonic mapping in $\mathbb{D}$. Then $\Lambda_{f}(z) \leq M \omega(1 / d(z))$ in $\mathbb{D}$ if and only if, for every $r \in(0,1-|z|]$,

$$
\frac{1}{|\mathbb{D}(z, r)|} \int_{\mathbb{D}(z, r)}|f(\zeta)-f(z)| d A(\zeta) \leq \operatorname{Mr} \omega\left(\frac{1}{r}\right),
$$

where $d A$ denotes the area measure in $\mathbb{D}$.

Definition 1.2. Let $f$ be harmonic in $\mathbb{D}$. For $p \in[1, \infty)$, we say that $f \in B M O_{p}$ if

$$
\|f\|_{B M O_{p}}=\sup _{\mathbb{D}(z, r) \subseteq \mathbb{D}}\left(\frac{1}{|\mathbb{D}(z, r)|} \int_{\mathbb{D}(z, r)}\left|f(\zeta)-\frac{1}{|\mathbb{D}(z, r)|} \int_{\mathbb{D}(z, r)} f(\xi) d A(\xi)\right|^{p} d A(\zeta)\right)^{1 / p}
$$

is bounded, where $r \in(0,1-|z|]$. 
In particular, by taking $\omega(t)=t$ in Theorem 1.1, we get the following result.

Corollary 1.3. Let $f$ be a harmonic mapping in $\mathbb{D}$. Then $f \in B M O_{1}$ if and only if $\Lambda_{f}(z) \leq M / d(z)$ holds in $\mathbb{D}$.

In [14], Dyakonov also investigated the property of equivalent modulus for holomorphic functions in $\mathbb{D}$ and obtained the following theorem.

Theorem B [14, Theorem 2]. Let $\omega$ be a regular majorant and $f$ be a holomorphic function in $\mathbb{D}$ and continuous up to the boundary $\partial \mathbb{D}$. Then

$$
f \in L_{\omega}(\mathbb{D}) \Longleftrightarrow|f| \in L_{\omega}(\mathbb{D}) \Longleftrightarrow|f| \in L_{\omega}(\mathbb{D}, \partial \mathbb{D}),
$$

where $L_{\omega}(\mathbb{D}, \partial \mathbb{D})$ denotes the class of continuous functions $F$ on $\mathbb{D} \cup \partial \mathbb{D}$ which satisfy (1.1) with some positive constant $C$, whenever $z \in \mathbb{D}$ and $w \in \partial \mathbb{D}$.

Later, in [28, Theorems A], Pavlović came up with a relatively simple proof of the results of Dyakonov. Recently, many authors have considered this topic and generalised Dyakonov's results to quasiconformal mappings and real harmonic functions in several variables for some special majorant $\omega(t)=t^{\alpha}$, where $\alpha>0$ (see $[1,15,23,26,27,29-31])$. For the general majorant $\omega$ to holomorphic mappings and pluriharmonic mappings in the unit ball, see [7, 15, 33].

We will prove the analogue of Theorem $B$ for real harmonic functions in the following form.

Theorem 1.4. Suppose that $\omega$ is a majorant satisfying (1.2), and that $G$ is an $L_{\omega^{-}}$ extension domain. If $f$ is a real harmonic function in $G$ and continuous up to the boundary $\partial G$, then

$$
f \in L_{\omega}(G) \Longleftrightarrow|f| \in L_{\omega}(G) \Longleftrightarrow|f| \in L_{\omega}(G, \partial G),
$$

where $L_{\omega}(G, \partial G)$ denotes the class of continuous functions $F$ on $G \cup \partial G$ which satisfy (1.1) with some positive constant $C$, whenever $z \in G$ and $w \in \partial G$.

Here a proper subdomain $G$ of $\mathbb{C}$ or $\mathbb{R}^{2}$ is said to be an $L_{\omega}$-extension if $L_{\omega}(G)=$ $\operatorname{loc} L_{\omega}(G)$, where $\operatorname{loc} L_{\omega}(G)$ denotes the set of all functions $f: G \rightarrow \mathbb{C}$ satisfying (1.1) with a fixed positive constant $M$, whenever $z \in G$ and $w \in G$ such that $|z-w|<\frac{1}{2} d_{G}(z)$. Obviously, the unit disc $\mathbb{D}$ is an $L_{\omega}$-extension domain.

In [25], the author proved that $G$ is an $L_{\omega}$-extension domain if and only if each pair of points $z, w \in G$ can be joined by a rectifiable curve $\gamma \subset G$ satisfying

$$
\int_{\gamma} \frac{\omega\left(d_{G}(z)\right)}{d_{G}(z)} d s(z) \leq M \omega(|z-w|)
$$

with some fixed positive constant $M=M(G, \omega)$, where $d s$ stands for the arc length measure on $\gamma$. See $[17,25]$ for more details on $L_{\omega}$-extension domains.

We remark that in Theorem 1.4, we replace 'the unit disc $\mathbb{D}$ ' and 'the regular majorant' in Theorem B by 'an $L_{\omega}$-extension domain' and 'a majorant satisfying (1.2), but not necessarily (1.3)', respectively. In fact, by using [30, Lemma A, Theorem 4, 
and Corollary 2] and the similar proof method of Theorem 1.4, we can prove that Theorem 1.4 also holds for real harmonic functions in the unit ball $\mathbb{B}^{n}$ of $\mathbb{R}^{n}$.

For planar harmonic mappings, we obtain the following result which is a generalisation of Theorem B.

THEOREM 1.5. Let $\omega$ be a majorant satisfying (1.2) and $G$ be an $L_{\omega}$-extension domain. Let $f=h+\bar{g}$ be a harmonic mapping in $G$, where $g$ and h are analytic functions in $G$. Then

$$
f \in L_{\omega}(G) \Longleftrightarrow g, h \in L_{\omega}(G) \Longleftrightarrow|g|,|h| \in L_{\omega}(G) .
$$

Definition 1.6. A planar harmonic mapping $f$ in $\mathbb{D}$ is called a harmonic Bloch mapping if

$$
\beta_{f}=\sup _{z, w \in \mathbb{D}, z \neq w} \frac{|f(z)-f(w)|}{\rho(z, w)}<\infty .
$$

Here $\beta_{f}$ is called the Lipschitz number of $f$ and

$$
\rho(z, w)=\frac{1}{2} \log \left(\frac{1+\left|\frac{z-w}{1-\bar{z} w}\right|}{1-\left|\frac{z-w}{1-\bar{z} w}\right|}\right)=\operatorname{arctanh}\left|\frac{z-w}{1-\bar{z} w}\right|
$$

denotes the hyperbolic distance between $z$ and $w$ in $\mathbb{D}$.

It is known that

$$
\beta_{f}=\sup _{z \in \mathbb{D}}\left\{\left(1-|z|^{2}\right) \Lambda_{f}(z)\right\}
$$

Clearly, a harmonic Bloch mapping $f$ is uniformly continuous as a map between metric spaces

$$
f:(\mathbb{D}, \rho) \rightarrow(\mathbb{C},|\cdot|)
$$

and for all $z, w \in \mathbb{D}$ we have the Lipschitz inequality

$$
|f(z)-f(w)| \leq \beta_{f} \rho(z, w) .
$$

The reader is referred to [12, Theorem 2] (or [2, 3, 8]) for a proof. Then the set of all harmonic Bloch mappings in $\mathbb{D}$ forms a harmonic Bloch space which is denoted by $\mathcal{B}_{h}$. Uniform continuity with respect to a hyperbolic metric is a central theme in $[35,36]$.

In [9, 20, 32], the authors provided several characterisations of $\mathrm{BMO}_{2}$ on holomorphic functions. For extensive discussions on $B M O_{2}$, see [11, 16, 18, 21, 24]. In this paper, we will use the $B M O_{2}$ norm to obtain a sharp estimate on harmonic Bloch mappings, which shows that $\mathcal{B}_{h}$ is isomorphic to $\mathrm{BMO}_{2}$ as a Banach space. Our result is given below.

Theorem 1.7. If $f$ is harmonic in $\mathbb{D}$, then

$$
\|f\|_{B M O_{2}} \leq \beta_{f} \leq 2\|f\|_{B M O_{2}} .
$$

Moreover, the estimates of (1.5) are sharp. The extreme harmonic mappings of the first inequality are constant functions, and the extreme harmonic mappings of the second inequality are the mappings with the form $f(z)=C(z+\bar{z})$, where $C$ denotes a constant. 
The proofs of Theorems 1.1 and 1.4 will be presented in Section 2, and the proof of Theorem 1.7 will be given in Section 3 .

\section{Bounded mean oscillation and equivalent modulus}

The following lemma easily follows from a simple computation (as in [6]).

LEMMa 2.1. Let $f$ be a complex-valued continuously differentiable function defined on $\mathbb{D}$ and $f=u+i v$, where $u$ and $v$ are real-valued functions. Then for $z=x+i y \in \mathbb{D}$,

$$
\Lambda_{f}(z) \leq|\nabla u(x, y)|+|\nabla v(x, y)|,
$$

where $\nabla u=\left(u_{x}, u_{y}\right)$ and $\nabla v=\left(v_{x}, v_{y}\right)$.

Then we have the following lemma.

Lemma 2.2. Suppose that $f$ is a harmonic mapping in $\overline{\mathbb{D}}(a, r)$, where $r$ is a positive constant. Then

$$
\Lambda_{f}(a) \leq \frac{2}{\pi r} \int_{0}^{2 \pi}\left|f(a)-f\left(a+r e^{i \theta}\right)\right| d \theta .
$$

PRoof. Let $f=u+i v$ be a harmonic mapping in $\overline{\mathbb{D}}(a, r)$, where $u$ and $v$ are real harmonic functions. Without loss of generality, we may assume that $a=0$ and $f(0)=0$. By Poisson's formula,

$$
u(z)=\frac{1}{2 \pi} \int_{0}^{2 \pi} \frac{r^{2}-|z|^{2}}{\left|z-r e^{i \theta}\right|^{2}} u\left(r e^{i \theta}\right) d \theta, \quad|z|<r .
$$

By calculations, we get $(z=x=i y)$

$$
u_{x}(z)=\frac{1}{2 \pi} \int_{0}^{2 \pi} \frac{-2 x\left|z-r e^{i \theta}\right|^{2}-2\left(r^{2}-|z|^{2}\right)(x-r \cos \theta)}{\left|z-r e^{i \theta}\right|^{4}} u\left(r e^{i \theta}\right) d \theta
$$

and similarly

$$
u_{y}(z)=\frac{1}{2 \pi} \int_{0}^{2 \pi} \frac{-2 y\left|z-r e^{i \theta}\right|^{2}-2\left(r^{2}-|z|^{2}\right)(y-r \sin \theta)}{\left|z-r e^{i \theta}\right|^{4}} u\left(r e^{i \theta}\right) d \theta,
$$

which imply that

$$
\begin{aligned}
|\nabla u(0)| & =\left(\left|\frac{1}{r \pi} \int_{0}^{2 \pi} u\left(r e^{i \theta}\right) \cos \theta d \theta\right|^{2}+\left|\frac{1}{r \pi} \int_{0}^{2 \pi} u\left(r e^{i \theta}\right) \sin \theta d \theta\right|^{2}\right)^{1 / 2} \\
& \leq \frac{1}{r \pi} \int_{0}^{2 \pi}(|\cos \theta|+|\sin \theta|)\left|u\left(r e^{i \theta}\right)\right| d \theta \\
& \leq \frac{\sqrt{2}}{r \pi} \int_{0}^{2 \pi}\left|u\left(r e^{i \theta}\right)\right| d \theta .
\end{aligned}
$$

A similar argument shows that

$$
|\nabla v(0)| \leq \frac{\sqrt{2}}{r \pi} \int_{0}^{2 \pi}\left|v\left(r e^{i \theta}\right)\right| d \theta .
$$


By (2.1), (2.2) and Lemma 2.1,

$$
\begin{aligned}
\Lambda_{f}(0) & \leq|\nabla u(0)|+|\nabla v(0)| \\
& \leq \frac{\sqrt{2}}{r \pi} \int_{0}^{2 \pi}\left(\left|u\left(r e^{i \theta}\right)\right|+\left|v\left(r e^{i \theta}\right)\right|\right) d \theta \\
& \leq \frac{2}{r \pi} \int_{0}^{2 \pi}\left|f\left(r e^{i \theta}\right)\right| d \theta .
\end{aligned}
$$

Finally, the desired conclusion follows if we apply the last inequality to the function $F(z)=f(a)-f(z+a)$.

2.1. Proof of Theorem 1.1. First, we show the 'if' part. By Lemma 2.2,

$$
\Lambda_{f}(z) \leq \frac{2}{\pi \rho} \int_{0}^{2 \pi}\left|f(z)-f\left(z+\rho e^{i \theta}\right)\right| d \theta
$$

where $\rho \in(0, d(z)]$, which gives

$$
\int_{0}^{r} \Lambda_{f}(z) \rho^{2} d \rho \leq \frac{2}{\pi} \int_{0}^{r}\left(\rho \int_{0}^{2 \pi}\left|f(z)-f\left(z+\rho e^{i \theta}\right)\right| d \theta\right) d \rho,
$$

whence

$$
\begin{aligned}
\Lambda_{f}(z) & \leq \frac{6}{\pi r^{3}} \int_{\mathbb{D}(z, r)}|f(z)-f(\zeta)| d A(\zeta) \\
& =\frac{6}{r|\mathbb{D}(z, r)|} \int_{\mathbb{D}(z, r)}|f(z)-f(\zeta)| d A(\zeta) \\
& \leq \frac{6 M k(r)}{r}=6 M \omega\left(\frac{1}{d(z)}\right),
\end{aligned}
$$

where $r=d(z)$.

Next, we prove the 'only if' part. For $z, w \in \mathbb{D}$ and $t \in(0,1)$,

$$
d(z+t(w-z))=1-|z+t(w-z)| \geq d(z)-t|w-z| .
$$

If $d(z)-t|w-z|>0$, then

$$
\begin{aligned}
|f(z)-f(w)| & \leq\left|\int_{0}^{1} \frac{d f}{d t}(z+t(w-z)) d t\right| \\
& \leq|w-z| \int_{0}^{1} \Lambda_{f}(z+t(w-z)) d t \\
& \leq M|w-z| \int_{0}^{1} \omega\left(\frac{1}{d(z)-t|w-z|}\right) d t \\
& =M \int_{0}^{|w-z|} \omega\left(\frac{1}{d(z)-t}\right) d t .
\end{aligned}
$$


Hence

$$
\begin{aligned}
\frac{1}{|\mathbb{D}(z, r)|} \int_{\mathbb{D}(z, r)}|f(\zeta)-f(z)| d A(\zeta) & \leq \frac{M}{\left|\mathbb{D}_{r}\right|} \int_{\mathbb{D}_{r}}\left(\int_{0}^{|\xi|} \omega\left(\frac{1}{d(z)-t}\right) d t\right) d A(\xi) \\
& =\frac{2 M}{r^{2}} \int_{0}^{r} \rho\left(\int_{0}^{\rho} \omega\left(\frac{1}{d(z)-t}\right) d t\right) d \rho \\
& \leq \frac{2 M}{r^{2}} \int_{0}^{r}\left(\int_{t}^{r} \rho d \rho\right) \omega\left(\frac{1}{r-t}\right) d t \\
& \leq \frac{2 M}{r} \int_{0}^{r}(r-t) \omega\left(\frac{1}{r-t}\right) d t \\
& \leq \frac{2 M}{r} r \omega\left(\frac{1}{r}\right) \int_{0}^{r} d t \\
& =2 M r \omega\left(\frac{1}{r}\right)
\end{aligned}
$$

The proof of this theorem is complete.

The following result from [22] is needed in the proof of Theorem 1.4.

Lemma C [22, Theorem 1]. Let $u$ be a real harmonic function of $\mathbb{D}$ into $(-1,1)$. Then for $z \in \mathbb{D}$, the following sharp inequality holds:

$$
|\nabla u(z)| \leq \frac{4}{\pi} \frac{1-u^{2}(z)}{1-|z|^{2}} .
$$

2.2. Proof of Theorem 1.4. Without loss of generality, we assume that $f$ is not constant. The implication $f \in L_{\omega}(G) \Rightarrow|f| \in L_{\omega}(G) \Rightarrow|f| \in L_{\omega}(G, \partial G)$ is obvious, and so we only need to prove the implication $|f| \in L_{\omega}(G) \Rightarrow f \in L_{\omega}(G)$. For a fixed $z \in G$, let

$$
M_{z}=\sup \left\{|f(\zeta)|:|\zeta-z|<d_{G}(z)\right\}
$$

and for $\xi \in \mathbb{D}$,

$$
T_{f}(\xi)=\frac{f\left(z+d_{G}(z) \xi\right)}{M_{z}} .
$$

Obviously, $\left|T_{f}(\xi)\right|<1$ and thus Lemma $C$ implies that

$$
\left|\nabla T_{f}(\xi)\right| \leq \frac{4}{\pi}\left(\frac{1-T_{f}^{2}(\xi)}{1-|\xi|^{2}}\right)
$$

which gives

$$
\frac{d_{G}(z)|\nabla f(z)|}{M_{z}}=\left|\nabla T_{f}(0)\right| \leq \frac{4}{\pi}\left(1-\frac{f^{2}(z)}{M_{z}^{2}}\right) \leq \frac{8}{\pi}\left(1-\frac{|f(z)|}{M_{z}}\right),
$$

that is,

$$
d_{G}(z)|\nabla f(z)| \leq \frac{8}{\pi}\left(M_{z}-|f(z)|\right)
$$


For a fixed $\varepsilon_{0}>0$, there exists a $\zeta \in \partial G$ such that $|\zeta-z|<\left(1+\varepsilon_{0}\right) d_{G}(z)$. Then, for $w \in \mathbb{D}\left(z, d_{G}(z)\right)$,

$$
\begin{aligned}
|f(w)|-|f(z)| & \leq\|f(w)|-| f(\zeta)\|+\|f(\zeta)|-| f(z)\| \\
& \leq M \omega\left(\left(2+\varepsilon_{0}\right) d_{G}(z)\right)+M \omega\left(\left(1+\varepsilon_{0}\right) d_{G}(z)\right) .
\end{aligned}
$$

Now we take $\varepsilon_{0}=1$. It follows that

$$
\sup _{w \in \mathbb{D}\left(z, d_{G}(z)\right)}(|f(w)|-|f(z)|) \leq M\left(\omega\left(3 d_{G}(z)\right)+\omega\left(2 d_{G}(z)\right)\right) \leq 5 M \omega\left(d_{G}(z)\right)
$$

whence

$$
M_{z}-|f(z)| \leq 5 M \omega\left(d_{G}(z)\right) .
$$

By (2.3) and (2.4), we conclude that

$$
|\nabla f(z)| \leq \frac{40 M}{\pi} \frac{\omega\left(d_{G}(z)\right)}{d_{G}(z)} .
$$

Finally, for $z_{1}, z_{2} \in G$, by [25], there must exist a rectifiable curve $\gamma$ in $G$ which joins $z_{1}$ and $z_{2}$ and satisfies (1.4). Integrating (2.5) along $\gamma$,

$$
\left|f\left(z_{1}\right)-f\left(z_{2}\right)\right| \leq \int_{\gamma}|\nabla f(\zeta)| d s(z) \leq \frac{40 M}{\pi} \int_{\gamma} \frac{\omega\left(d_{G}(z)\right)}{d_{G}(z)} d s(z) \leq C \omega\left(\left|z_{1}-z_{2}\right|\right),
$$

where $C$ is a constant. The proof of this theorem is complete.

Proof of Theorem 1.5. The implication $g, h \in L_{\omega}(G) \Longleftrightarrow|g|,|h| \in L_{\omega}(G)$ follows from Theorem B. We only need to prove that $f \in L_{\omega}(G) \Longrightarrow g, h \in L_{\omega}(G)$, because the implication $g, h \in L_{\omega}(G) \Longrightarrow f \in L_{\omega}(G)$ is obvious. Let $f=h+\bar{g}$ in $G$, where $h$ and $g$ are holomorphic in $G$. It is easy to see that $f \in L_{\omega}(G) \Longrightarrow \bar{f} \in L_{\omega}(G)$. This implies that $u=\operatorname{Re} f_{1} \in L_{\omega}(G)$ and $v=\operatorname{Im} f_{2} \in L_{\omega}(G)$, where $f_{1}=h+g$ and $f_{2}=h-g$.

We claim that $f_{1}, f_{2} \in L_{\omega}(G)$. We now prove this claim. For a fixed $z \in G$, let

$$
M_{z}=\sup \{|u(\zeta)|:|\zeta-z|<d(z)\} \quad \text { and } \quad T_{u}(\xi)=\frac{u(z+d(z) \xi)}{M_{z}}, \quad \xi \in \mathbb{D} .
$$

Then for any $\xi \in \mathbb{D},\left|T_{u}(\xi)\right|<1$ and by Lemma $\mathrm{C}$,

$$
\left|\nabla T_{u}(\xi)\right| \leq \frac{4}{\pi}\left(\frac{1-T_{u}^{2}(\xi)}{1-|\xi|^{2}}\right)
$$

This gives

$$
\frac{d(z)|\nabla u(z)|}{M_{z}}=\left|\nabla T_{u}(0)\right| \leq \frac{4}{\pi}\left(1-\frac{u^{2}(z)}{M_{z}^{2}}\right) \leq \frac{8}{\pi}\left(1-\frac{|u(z)|}{M_{z}}\right)
$$

which yields

$$
d(z)\left|f_{1}^{\prime}(z)\right|=d(z)|\nabla u(z)| \leq \frac{8}{\pi}\left(M_{z}-|u(z)|\right)
$$


For a fixed $\varepsilon_{0}>0$, there exists a $\zeta \in \partial G$ such that $|\zeta-z|<\left(1+\varepsilon_{0}\right) d_{G}(z)$. Then, for $w \in \mathbb{D}\left(z, d_{G}(z)\right)$,

$$
\begin{aligned}
|u(w)|-|u(z)| & \leq\|u(w)|-| u(\zeta)\|+\|u(\zeta)|-| u(z)\| \\
& \leq M \omega\left(\left(2+\varepsilon_{0}\right) d_{G}(z)\right)+M \omega\left(\left(1+\varepsilon_{0}\right) d_{G}(z)\right) .
\end{aligned}
$$

Now we take $\varepsilon_{0}=1$. It follows that

$$
\sup _{w \in \mathbb{D}\left(z, d_{G}(z)\right)}(|u(w)|-|u(z)|) \leq M\left(\omega\left(3 d_{G}(z)\right)+\omega\left(2 d_{G}(z)\right)\right) \leq 5 M \omega\left(d_{G}(z)\right)
$$

whence

$$
M_{z}-|u(z)| \leq 5 M \omega\left(d_{G}(z)\right) .
$$

By (2.6) and (2.7), we conclude that

$$
\left|f_{1}^{\prime}(z)\right| \leq \frac{40 M}{\pi} \frac{\omega\left(d_{G}(z)\right)}{d_{G}(z)} .
$$

Finally, for $z_{1}, z_{2} \in G$, by [25], there must exist a rectifiable curve $\gamma$ in $G$ which joins $z_{1}$ and $z_{2}$, and satisfies (1.4). Integrating (2.8) along $\gamma$, we obtain that

$$
\left|f_{1}\left(z_{1}\right)-f_{1}\left(z_{2}\right)\right| \leq \int_{\gamma}\left|f_{1}^{\prime}(\zeta)\right| d s(z) \leq \frac{40 M}{\pi} \int_{\gamma} \frac{\omega\left(d_{G}(z)\right)}{d_{G}(z)} d s(z) \leq C \omega\left(\left|z_{1}-z_{2}\right|\right),
$$

where $C$ is a constant. This gives $f_{1} \in L_{\omega}(G)$. By similar arguments, we know that $f_{2} \in L_{\omega}(G)$. Hence $\left(f_{1}+f_{2}\right) \in L_{\omega}(G)$ and $\left(f_{1}-f_{2}\right) \in L_{\omega}(G)$. Therefore,

$$
h=\frac{f_{1}+f_{2}}{2} \in L_{\omega}(G) \quad \text { and } \quad g=\frac{f_{1}-f_{2}}{2} \in L_{\omega}(G) .
$$

The proof of this theorem is complete.

\section{Estimates on $\mathrm{BMO}_{2}$}

Green's theorem (see $[4,5])$ states that if $g \in C^{2}(\mathbb{D})$, that is, is twice continuously differentiable in $\mathbb{D}$, then

$$
\frac{1}{2 \pi} \int_{0}^{2 \pi} g\left(r e^{i \theta}\right) d \theta=g(0)+\frac{1}{2} \int_{\mathbb{D}_{r}} \Delta g(z) \log \frac{r}{|z|} d \sigma(z)
$$

for $r \in(0,1)$, where $d \sigma$ denotes the normalised area measure in $\mathbb{D}$.

LeMma 3.1. For $r \in(0,1)$, let

$$
M_{p}^{p}(r, f)=\frac{1}{2 \pi} \int_{0}^{2 \pi}\left|f\left(r e^{i \theta}\right)\right|^{p} d \theta
$$


where $f$ is a harmonic mapping in $\mathbb{D}$. Then for $p \in[2, \infty), M_{p}^{p}(r, f)$ is an increasing function on $r$ in $(0,1)$ and

$$
\begin{aligned}
r \frac{d}{d r} M_{p}^{p}(r, f)= & p \int_{\mathbb{D}_{r}}\left(\left(\frac{p}{2}-1\right)|f(z)|^{p-4}\left|f_{z}(z) \overline{f(z)}+f(z) \overline{f_{\bar{z}}(z)}\right|^{2}\right. \\
& \left.+|f(z)|^{p-2}|\widehat{\nabla f}(z)|^{2}\right) d \sigma(z),
\end{aligned}
$$

where $|\widehat{\nabla f}|=\left(\left|f_{z}\right|^{2}+\left|f_{\bar{z}}\right|^{2}\right)^{1 / 2}$.

Proof. Since $|f|^{p}$ is subharmonic in $\mathbb{D}$, we see that $M_{p}^{p}(r, f)$ is an increasing function on $r$ in $(0,1)$, where $p \in[2, \infty)$. On the other hand, by $(3.1)$,

$$
\begin{aligned}
r \frac{d}{d r} M_{p}^{p}(r, f)= & \frac{1}{2} \int_{\mathbb{D}_{r}} \Delta\left(|f(z)|^{p}\right) d \sigma(z) \\
= & p \int_{\mathbb{D}_{r}}\left(\left(\frac{p}{2}-1\right)|f(z)|^{p-4}\left|f_{z}(z) \overline{f(z)}+f(z) \overline{f_{\bar{z}}(z)}\right|^{2}\right. \\
& \left.+|f(z)|^{p-2}|\widehat{\nabla f}(z)|^{2}\right) d \sigma(z) .
\end{aligned}
$$

The proof of this lemma is complete.

Lemma 3.2. For $r \in(0,1)$ and $p \in[2, \infty)$, let

$$
I_{p}(r, f)=\left(\frac{1}{\left|\mathbb{D}_{r}\right|} \int_{\mathbb{D}_{r}}|f(z)|^{p} d A(z)\right)^{1 / p},
$$

where $f$ is harmonic in $\mathbb{D}$. Then the function $I_{p}(r, f)$ is increasing on $r$ in $(0,1)$.

Proof. Since

$$
\int_{\mathbb{D}_{r}}|f(z)|^{p} d A(z)=2 \pi \int_{0}^{r} \rho M_{p}^{p}(\rho, f) d \rho,
$$

we see that

$$
\frac{d}{d r} \int_{\mathbb{D}_{r}}|f(z)|^{p} d A(z)=2 \pi r M_{p}^{p}(r, f) .
$$

By (3.2), (3.3) and Lemma 3.1,

$$
M_{p}^{p}(r, f)-I_{p}^{p}(r, f)=\frac{1}{\left|\mathbb{D}_{r}\right|} \int_{0}^{r} \frac{d}{d t} M_{p}^{p}(t, f)\left|\mathbb{D}_{t}\right| d t \geq 0 .
$$

By (3.2), (3.4) and elementary computations,

$$
\begin{aligned}
\frac{d}{d r} I_{p}^{p}(r, f) & =\frac{\left|\mathbb{D}_{r}\right| \frac{d}{d r} \int_{\mathbb{D}_{r}}|f(z)|^{p} d A(z)-\int_{\mathbb{D}_{r}}|f(z)|^{p} d A(z) \frac{d}{d r}\left|\mathbb{D}_{r}\right|}{\left|\mathbb{D}_{r}\right|^{2}} \\
& =\frac{2 \pi r\left[\left|\mathbb{D}_{r}\right| M_{p}^{p}(r, f)-\int_{\mathbb{D}_{r}}|f(z)|^{p} d A(z)\right]}{\left|\mathbb{D}_{r}\right|^{2}} \\
& \geq 0
\end{aligned}
$$


Hence the function $I_{p}(r, f)$ is increasing on $r$ in $(0,1)$. The proof of this lemma is complete.

Lemma 3.3. For fixed $a \in \mathbb{D}$, let $\phi_{a}(z)=a+(1-|a|) z$ in $\mathbb{D}$. Then for $p \in[2, \infty)$,

$$
\|f\|_{B M O_{p}}=\sup _{a \in \mathbb{D}}\left(\frac{1}{|\mathbb{D}|} \int_{\mathbb{D}}\left|f\left(\phi_{a}(z)\right)-f\left(\phi_{a}(0)\right)\right|^{p} d A(z)\right)^{1 / p},
$$

where $f$ is harmonic in $\mathbb{D}$.

Proof. It is not difficult to see that

$$
\sup _{a \in \mathbb{D}}\left(\frac{1}{|\mathbb{D}|} \int_{\mathbb{D}}\left|f\left(\phi_{a}(z)\right)-f\left(\phi_{a}(0)\right)\right|^{p} d A(z)\right)^{1 / p} \leq\|f\|_{B M O_{p}} .
$$

On the other hand, by elementary calculations and Lemma 3.2,

$$
\begin{aligned}
& \left(\frac{1}{|\mathbb{D}(a, r)|} \int_{\mathbb{D}(a, r)}|f(\zeta)-f(a)|^{p} d A(\zeta)\right)^{1 / p} \\
& \quad \leq\left(\frac{1}{|\mathbb{D}(a, 1-|a|)|} \int_{\mathbb{D}(a, 1-|a|)}|f(\zeta)-f(a)|^{p} d A(\zeta)\right)^{1 / p} \\
& \quad=\left(\frac{1}{|\mathbb{D}|} \int_{\mathbb{D}}\left|f\left(\phi_{a}(\zeta)\right)-f\left(\phi_{a}(0)\right)\right|^{p} d A(\zeta)\right)^{1 / p}
\end{aligned}
$$

where $r \in(0,1-|a|]$. Then

$$
\|f\|_{B M O_{p}} \leq \sup _{a \in \mathbb{D}}\left(\frac{1}{|\mathbb{D}|} \int_{\mathbb{D}}\left|f\left(\phi_{a}(z)\right)-f\left(\phi_{a}(0)\right)\right|^{p} d A(z)\right)^{1 / p} .
$$

Obviously, (3.5) follows from (3.6) and (3.7).

Lemma 3.4. For each fixed $a \in \mathbb{D}$, let $\phi_{a}(z)=a+(1-|a|) z$ in $\mathbb{D}$. Then

$$
\left|\phi_{a}^{\prime}(z)\right| \leq \frac{1-\left|\phi_{a}(z)\right|^{2}}{1-|z|^{2}} .
$$

Proof. It is easy to see that $f$ is analytic and, for all $z \in \mathbb{D},\left|\phi_{a}(z)\right| \leq 1$. Then (3.8) follows from the Schwarz-Pick lemma.

3.1. Proof of Theorem 1.7. We first prove that $\|f\|_{B M O_{2}} \leq \beta_{f}$. For a fixed $a \in \mathbb{D}$, let

$$
F_{a}(\zeta)=f\left(\phi_{a}(\zeta)\right)
$$

in $\mathbb{D}$, where $\phi_{a}(\zeta)=a+(1-|a|) \zeta$. By Lemma 3.4,

$$
\begin{aligned}
\sup _{\zeta \in \mathbb{D}}\left(\left(1-|\zeta|^{2}\right) \Lambda_{F_{a}}(\zeta)\right) & =\sup _{\zeta \in \mathbb{D}}\left(\left(1-|\zeta|^{2}\right) \Lambda_{f}\left(\phi_{a}(\zeta)\right)\left|\phi_{a}^{\prime}(\zeta)\right|\right) \\
& \leq \sup _{\zeta \in \mathbb{D}}\left(\left(1-\left|\phi_{a}(\zeta)\right|^{2}\right) \Lambda_{f}\left(\phi_{a}(\zeta)\right)\right) \\
& \leq \beta_{f} .
\end{aligned}
$$


Then Lemma 3.1 leads to

$$
\begin{aligned}
\frac{d}{d r} M_{2}^{2}\left(r, F_{a}\left(r e^{i \theta}\right)-F_{a}(0)\right) & =\frac{2}{r \pi} \int_{\mathbb{D}_{r}}\left|\widehat{\nabla F_{a}}(\zeta)\right|^{2} d A(\zeta) \\
& \leq \frac{2}{r \pi} \int_{\mathbb{D}_{r}} \Lambda_{F_{a}}^{2}(\zeta) d A(\zeta) \\
& \leq \frac{2 \beta_{f}^{2}}{r \pi} \int_{\mathbb{D}_{r}} \frac{d A(\zeta)}{\left(1-|\zeta|^{2}\right)^{2}} \\
& =\frac{4 \beta_{f}^{2}}{r} \int_{0}^{r} \frac{\rho}{\left(1-\rho^{2}\right)^{2}} d \rho \\
& =2 \beta_{f}^{2} \sum_{n=1}^{\infty} r^{2 n-1},
\end{aligned}
$$

which gives

$$
M_{2}^{2}\left(r, F_{a}\left(r e^{i \theta}\right)-F_{a}(0)\right) \leq \beta_{f}^{2} \sum_{n=1}^{\infty} \frac{r^{2 n}}{n}
$$

Since

$$
\begin{aligned}
\int_{0}^{1} 2 r M_{2}^{2}\left(r, F_{a}\left(r e^{i \theta}\right)-F_{a}(0)\right) d r & =\frac{1}{\pi} \int_{0}^{1} \int_{0}^{2 \pi} r\left|F_{a}\left(r e^{i \theta}\right)-F_{a}(0)\right|^{2} d \theta d r \\
& =\frac{1}{|\mathbb{D}|} \int_{\mathbb{D}}\left|F_{a}(\zeta)-F_{a}(0)\right|^{2} d A(\zeta),
\end{aligned}
$$

we see that

$$
\frac{1}{|\mathbb{D}|} \int_{\mathbb{D}}\left|F_{a}(\zeta)-F_{a}(0)\right|^{2} d A(\zeta) \leq \int_{0}^{1} 2 \beta_{f}^{2} \sum_{n=1}^{\infty} \frac{r^{2 n+1}}{n} d r=\beta_{f}^{2} \sum_{n=1}^{\infty} \frac{1}{n(n+1)}=\beta_{f}^{2},
$$

whence

$$
\|f\|_{B M O_{2}} \leq \beta_{f} .
$$

Next, we prove that $\beta_{f} \leq 2\|f\|_{B M O_{2}}$. By Lemma 3.1 and the subharmonicity of $\left|\widehat{\nabla F_{a}}\right|^{2}$

$$
\begin{aligned}
\frac{2}{r} \int_{0}^{r} \rho\left|\widehat{\nabla F_{a}}(0)\right|^{2} d \rho & \leq \frac{2}{r} \int_{0}^{r} \rho\left(\frac{1}{2 \pi} \int_{0}^{2 \pi}\left|\widehat{\nabla F_{a}}\left(\rho e^{i \theta}\right)\right|^{2} d \theta\right) d \rho \\
& =\frac{1}{r \pi} \int_{\mathbb{D}_{r}}\left|\widehat{\nabla F_{a}}(\zeta)\right|^{2} d A(\zeta) \\
& =\frac{1}{2} \frac{d}{d r} M_{2}^{2}\left(r, F_{a}\left(r e^{i \theta}\right)-F_{a}(0)\right),
\end{aligned}
$$

which implies that

$$
\left|\widehat{\nabla F_{a}}(0)\right|^{2} r^{2} \leq M_{2}^{2}\left(r, F_{a}\left(r e^{i \theta}\right)-F_{a}(0)\right)
$$


It follows that

$$
\frac{\left|\widehat{\nabla F_{a}}(0)\right|^{2}}{4}=\int_{0}^{1}\left|\widehat{\nabla F_{a}}(0)\right|^{2} r^{3} d r \leq \frac{1}{2 \pi} \int_{\mathbb{D}}\left|F_{a}(\zeta)-F_{a}(0)\right|^{2} d A(\zeta),
$$

whence

$$
\frac{\Lambda_{F_{a}}^{2}(0)}{4} \leq \frac{\left|\widehat{\nabla F_{a}}(0)\right|^{2}}{2} \leq \frac{1}{|\mathbb{D}|} \int_{\mathbb{D}}\left|F_{a}(\zeta)-F_{a}(0)\right|^{2} d A(\zeta) .
$$

On the other hand,

$$
\beta_{f} \leq \sup _{a \in \mathbb{D}} \Lambda_{F_{a}}(0)
$$

By (3.9) and (3.10),

$$
\beta_{f} \leq 2\|f\|_{B M O_{2}} \text {. }
$$

It remains to prove the sharpness in the inequalities. Obviously, the equality sign in the first inequality of (1.5) occurs when $f$ is constant. For the sharpness part of the second inequality of (1.5), we let

$$
f(z)=C(z+\bar{z})
$$

where $C$ is a constant. Then

$$
\beta_{f}=\sup _{z \in \mathbb{D}}\left\{\left(1-|z|^{2}\right) \Lambda_{f}(z)\right\}=2|C|
$$

and

$$
\begin{aligned}
\|f\|_{B M O_{2}} & =\sup _{a \in \mathbb{D}}\left(\frac{1}{|\mathbb{D}|} \int_{\mathbb{D}}\left|F_{a}(z)-F_{a}(0)\right|^{2} d A(z)\right)^{1 / 2} \\
& =|C| \sup _{a \in \mathbb{D}}\left(\frac{1}{|\mathbb{D}|} \int_{\mathbb{D}}(1-|a|)^{2}|z+\bar{z}|^{2} d A(z)\right)^{1 / 2} \\
& =|C| \sup _{a \in \mathbb{D}}\left(\frac{4(1-|a|)^{2}}{\pi} \int_{0}^{1} \int_{0}^{2 \pi} r^{3} \cos ^{2} \theta d \theta d r\right)^{1 / 2} \\
& =|C| \sup _{a \in \mathbb{D}}(1-|a|) \\
& =|C|,
\end{aligned}
$$

whence

$$
\beta_{f}=2\|f\|_{B M O_{2}} .
$$

The proof of this theorem is complete.

\section{References}

[1] M. Arsenović, V. Božin and V. Manojlović, 'Moduli of continuity of harmonic quasiregular mappings in $\mathbb{B}^{n}$, Potential Anal. 34 (2011), 283-291.

[2] Sh. Chen, S. Ponnusamy and X. Wang, 'Bloch constant and Landau's theorems for planar $p$ harmonic mappings', J. Math. Anal. Appl. 373 (2011), 102-110.

[3] Sh. Chen, S. Ponnusamy and X. Wang, 'Landau's theorem and Marden constant for harmonic v-Bloch mappings', Bull. Aust. Math. Soc. 84 (2011), 19-32. 
[4] Sh. Chen, S. Ponnusamy and X. Wang, 'On planar harmonic Lipschitz and planar harmonic Hardy classes', Ann. Acad. Sci. Fenn. Math. 36 (2011), 567-576.

[5] Sh. Chen, S. Ponnusamy and X. Wang, 'Integral means and coefficient estimates on planar harmonic mappings', Ann. Acad. Sci. Fenn. Math. 37 (2012), 69-79.

[6] Sh. Chen, S. Ponnusamy and X. Wang, 'Area integral means, Hardy and weighted Bergman spaces on planar harmonic mappings', Kodai Math. J., to appear, http://arxiv.org/abs/1203.2711.

[7] Sh. Chen, S. Ponnusamy and X. Wang, 'Equivalent moduli of continuity, Bloch's theorem for pluriharmonic mappings in $\mathbb{B}^{n}$ ', Proc. Indian Acad. Sci. Math. Sci. 122(4) (2012), 583-595.

[8] Sh. Chen and X. Wang, 'On harmonic Bloch spaces in the unit ball of $\mathbb{C}^{n}$ ', Bull. Aust. Math. Soc. 84 (2011), 67-78.

[9] J. G. Clunie and T. H. MacGregor, 'Radial growth of the derivative of univalent functions', Comment. Math. Helv. 59 (1984), 362-375.

[10] J. G. Clunie and T. Sheil-Small, 'Harmonic univalent functions', Ann. Acad. Sci. Fenn. Ser. A I Math. 9 (1984), 3-25.

[11] R. R. Coifman, R. Rochberg and G. Weiss, 'Factorization theorems for Hardy spaces in several complex variables', Ann. of Math. (2) 103 (1976), 611-635.

[12] F. Colonna, 'The Bloch constant of bounded harmonic mappings', Indiana Univ. Math. J. 38 (1989), 829-840.

[13] P. Duren, Harmonic Mappings in the Plane (Cambridge University Press, Cambridge, 2004).

[14] K. M. Dyakonov, 'Equivalent norms on Lipschitz-type spaces of holomorphic functions', Acta Math. 178 (1997), 143-167.

[15] K. M. Dyakonov, 'Holomorphic functions and quasiconformal mappings with smooth moduli', Adv. Math. 187 (2004), 146-172.

[16] C. Fefferman and E. M. Stein, ' $H^{p}$ spaces of several variables', Acta Math. 129 (1972), 137-193.

[17] F. W. Gehring and O. Martio, 'Lipschitz-classes and quasiconformal mappings', Ann. Acad. Sci. Fenn. Ser. A I Math. 10 (1985), 203-219.

[18] K. T. Hahn and E. H. Youssfi, ' $\mathcal{M}$-harmonic Besov p-spaces and Hankel operators in the Bergman space on the ball in $\mathbb{C}^{n}$, Manuscripta Math. 71 (1991), 67-81.

[19] R. R. Hall, 'On an inequality of E. Heinz', J. Anal. Math. 42 (1982/83), 185-198.

[20] W. K. Hayman and Ch. Pommerenke, 'On analytic functions of bounded mean oscillation', Bull. Lond. Math. Soc. 10 (1978), 214-224.

[21] M. Jevtić, 'M-harmonic Bloch space and $B M O$ in the Bergman metric on the ball', Zb. Rad. 6 (1992), 83-88.

[22] D. Kalaj and M. Vuorinen, 'On harmonic functions and the Schwarz lemma', Proc. Amer. Math. Soc. 140 (2012), 161-165.

[23] V. Kojić and M. Pavlović, 'Subharmonicity of $|f|^{p}$ for quasiregular harmonic functions, with applications', J. Math. Anal. Appl. 342 (2008), 742-746.

[24] B. Korenblum, 'BMO estimates and radial growth of Bloch functions', Bull. Amer. Math. Soc. 12 (1985), 99-102.

[25] V. Lappalainen, 'Lip ${ }_{h}$-extension domains', Ann. Acad. Sci. Fenn. Ser. A I Math. Diss. 56 (1985).

[26] M. Mateljević, 'Quasiconformal and quasiregular harmonic analogues of Koebe's theorem and applications', Ann. Acad. Sci. Fenn. Math. 32 (2007), 301-315.

[27] M. Mateljević and M. Vuorinen, 'On harmonic quasiconformal quasi-isometries', J. Inequal. Appl. 2010, Article ID 178732, 19 pages, doi:10.1155/2010/1787.

[28] M. Pavlović, 'On Dyakonov's paper equivalent norms on Lipschitz-type spaces of holomorphic functions', Acta Math. 183 (1999), 141-143.

[29] M. Pavlović, Introduction to Function Spaces on the Disk, Matematički Institut SANU, Belgrade, 2004.

[30] M. Pavlović, 'Lipschitz conditions on the modulus of a harmonic function', Rev. Mat. Iberoam. 23 (2007), 831-1845.

[31] M. Pavlović, 'A Schwarz lemma for the modulus of a vector-valued analytic function', Proc. Amer. Math. Soc. 139 (2011), 969-973.

[32] Ch. Pommerenke, Boundary Behaviour of Conformal Maps (Springer, Berlin, 1992). 
[33] J. Qiao and X. Wang, Lipschitz-type spaces of pluriharmonic mappings, Filomat, to appear.

[34] T. Sheil-Small, 'Constants for planar harmonic mappings', J. Lond. Math. Soc. 42 (1990), 237-248.

[35] M. Vuorinen, Conformal Geometry and Quasiregular Mappings, Lecture Notes in Mathematics, 1319 (Springer, Berlin, 1988), 209pp.

[36] M. Vuorinen, 'Geometry of metrics', J. Anal. 18 (2010), 399-424.

SH. CHEN, Department of Mathematics and Computational Science, Hengyang Normal University, Hengyang, Hunan 421008,

PR China

e-mail: shlchen1982@yahoo.com.cn

S. PONNUSAMY, Indian Statistical Institute (ISI), Chennai Centre, SETS (Society for Electronic Transactions and Security), MGR Knowledge City, CIT Campus, Taramani, Chennai 600 113, India

e-mail: samy@iitm.ac.in, samy@isichennai.res.in

M. VUORINEN, Department of Mathematics, University of Turku, Turku 20014, Finland

e-mail: vuorinen@utu.fi

X. WANG, Department of Mathematics, Hunan Normal University, Changsha, Hunan 410081, PR China

e-mail: xtwang@hunnu.edu.cn 